\title{
The strong coupling and its running to four loops in a minimal MOM scheme
}

\author{
Lorenz von Smekal ${ }^{\mathrm{a}, \mathrm{b}}$, Kim Maltman ${ }^{\mathrm{a}, \mathrm{c}}$, André Sternbeck ${ }^{\mathrm{a}}$ \\ ${ }^{a}$ CSSM, School of Chemistry $\mathcal{E}$ Physics, The University of Adelaide, SA 5005, Australia \\ ${ }^{b}$ Institut für Kernphysik, Technische Universität Darmstadt, Schlossgartenstr. 9, D-64289 Darmstadt, Germany \\ ${ }^{c}$ Department of Mathematics and Statistics, York University, Toronto, ON, M3J 1P3, Canada
}

\begin{abstract}
We introduce the minimal momentum subtraction (MiniMOM) scheme for QCD. Its definition allows the strong coupling to be fixed solely through a determination of the gluon and ghost propagators. In Landau gauge this scheme has been implicit in the early studies of these propagators, especially in relation to their non-perturbative behaviour in the infrared and the associated infrared fixed-point. Here we concentrate on its perturbative use. We give the explicit perturbative definition of the scheme and the relation of its $\beta$-function and running coupling to the $\overline{\mathrm{MS}}$ scheme up to 4-loop order in general covariant gauges. We also demonstrate, by 'considering a selection of $N_{f}=3$ examples, that the apparent convergence of the relevant perturbative series can in some (though not all) cases be significantly improved by re-expanding the $\overline{\mathrm{MS}}$ coupling version of this series in terms of the MiniMOM coupling, making the MiniMOM coupling also of potential interest in certain phenomenological applications.
\end{abstract}

Key words: strong coupling constant, 4-loop running, minimal subtraction, momentum subtraction PACS: 12.38.Gc, 12.38.Aw, etc.

\section{Introduction}

The coupling constant of the strong interaction, $\alpha_{s}$, is one of the fundamental parameters of the Standard Model. It is specified by its value in a particular renormalisation scheme at a chosen reference scale, $\mu$, conventionally taken to be the $\overline{\mathrm{MS}}$ scheme at the $N_{f}=5$ reference scale $\mu=M_{Z}$. A recent assessment of experimental results yields $\alpha_{s}^{\overline{\mathrm{MS}}}\left(M_{Z}\right)=0.1189(10)$ [1]. This result, which is little changed if more recent experimental results are taken into account (see, e.g., [2] and references therein), is in excellent agreement with two recent, slightly different, lattice determinations [2, 3] based on lattice perturbation theory analyses of short-distance-sensitive lattice observables computed using the MILC $N_{f}=2+1$ configurations.

Other schemes than the $\overline{\mathrm{MS}}$ scheme are of course also possible. For example it has been proposed in [4] that a particular product of dimensionless gluon and ghost dressing functions, $Z$ and $G$, in the Landau gauge can be used to define a nonperturbative running coupling via

$$
\alpha_{s}^{\mathrm{MM}}\left(p^{2}\right)=\frac{g^{2}}{4 \pi} Z\left(p^{2}\right) G^{2}\left(p^{2}\right),
$$

where $g^{2} \equiv g^{2}(\mu)$ is the renormalised coupling at scale $\mu$ and the renormalisation condition,

$$
\left.Z\left(p^{2}\right) G^{2}\left(p^{2}\right)\right|_{p^{2}=\mu^{2}}=1,
$$

is assumed. The definition (1) is particularly convenient since it allows the coupling and hence $\Lambda^{\overline{\mathrm{MS}}}$ to be determined by measuring two-point functions on a lattice. Our first steps towards such a determination for $N_{f}=0,2$ were presented at the 2007
Lattice Conference [5]. Further updates of these preliminary results were reported last year [6, 7]. This project, which is ongoing, has the potential to provide an independent precision determination of $\alpha_{s}$ from lattice simulations at purely perturbative scales, typically between 10 and $100 \mathrm{GeV}$ [8] 1 As an important supplement we specify here the details of the renormalisation scheme underlying the coupling (1) for QCD in general covariant gauges. We also provide the explicit 4-loop $\beta$ function for $\alpha_{s}^{\mathrm{MM}}$ in all such gauges. This information will be important to the previously mentioned lattice analysis and to quantifying the truncation error on the resulting $\alpha_{s}$ determination.

The product in (1) is dimensionless and renormalisation group invariant, and it reduces to the running coupling of a perturbative momentum subtraction scheme (MOM) at large $p^{2}$. This makes it a suitable candidate for a non-perturbative extension, though such extensions are, of course, not unique. The underlying renormalisation condition (2) respects infrared scaling (with $0.5<\kappa<1[4]$ ),

$$
Z\left(p^{2}\right) \sim\left(p^{2} / \Lambda_{\mathrm{QCD}}^{2}\right)^{2 \kappa}, \quad \text { and } G\left(p^{2}\right) \sim\left(p^{2} / \Lambda_{\mathrm{QCD}}^{2}\right)^{-\kappa},
$$

for $p^{2} \rightarrow 0$, as predicted by a variety of functional continuum methods for Landau gauge QCD including studies of Dyson-Schwinger Equations (DSEs) [9], Stochastic Quantisation [10], and of the Functional Renormalisation Group Equations (FRGEs) [11]. This conformal infrared behaviour of the

\footnotetext{
${ }^{1}$ The QCD scale parameter of the underlying scheme (the MiniMOM scheme, see below) is roughly $450 \mathrm{MeV}$ for $N_{f}=0$, or $430 \mathrm{MeV}$ for $N_{f}=2$. Non-perturbative contributions to the gluon and ghost dressing functions are at least of the order $\left(\Lambda_{\mathrm{QCD}} / \mu\right)^{2}$. They are negligible at scales $\mu$ above $10 \mathrm{GeV}$.
} 
purely gluonic correlations in Landau gauge QCD is consistent with the conditions for confinement in local quantum field theory [12, 13], but it is yet to be observed in lattice simulations 2

If the infrared scaling behaviour (3) is realised, the renormalisation condition (2) holds beyond perturbation theory and can be imposed at any (space-like) subtraction point $p^{2}=\mu^{2} \geq 0$. This is because the running coupling defined by (1) then approaches a finite infrared fixed-point, $\alpha_{S}^{\mathrm{MM}} \rightarrow \alpha_{c}$ for $p^{2} \rightarrow 0$, with $\alpha_{c} \approx 8.9 / N_{c}$ obtained under a mild regularity assumption on the ghost-gluon vertex [9]. For the purposes of this Letter it suffices, however, that the coupling (1), is well-defined perturbatively, independent of the infrared scaling behaviour in (3).

A special feature of Landau gauge which underlies the definition (1) is the non-renormalisation of the ghost-gluon vertex [15]. The possibility of taking advantage of this nonrenormalisation has been criticised in the past [16, 17] on the grounds that the Landau-gauge ghost-gluon vertex acquires a momentum dependence in all common MOM schemes, already at one-loop level [18], despite the absence of ultraviolet divergences in this vertex in Landau gauge. There is, however, no contradiction here at all, as will be explained in the next section. The basic reason is that the non-renormalisation of the ghostgluon vertex ensures the existence of a scheme for which the notion of (1) as a running coupling makes sense and which this running coupling implicitly defines, without the need to use an asymmetric momentum scheme [19]. We call it the MiniMOM scheme for reasons that will become clear below. A useful feature of the MiniMOM scheme is that it can be related to the $\overline{\mathrm{MS}}$ scheme at four-loops [6, 7] without the need to compute vertices to three loops in perturbation theory.

After providing more details on the MiniMOM coupling and its relation to the $\overline{\mathrm{MS}}$ coupling below, we consider, in Sec. 4 , the $\overline{\mathrm{MS}}$ and MiniMOM versions of the perturbative series entering a selection of phenomenological applications, demonstrating that, in some cases, the apparent convergence of the series is much improved by the use of the MiniMOM coupling. Our conclusions and outlook are given in Sec.5.

\section{The minimal MOM scheme}

Some confusion in the literature concerning the running coupling of Eq. (1) arose in relation to the misconception that this definition rests on the non-renormalisation of the ghost-gluon vertex in Landau gauge [15]. This definition is not, however, based on requiring that the ghost-gluon vertex reduce to the tree-level one at a symmetric subtraction point $k^{2}=p^{2}=q^{2}=$ $\mu^{2}$. In particular, the scheme underlying (1) is not the MOMh scheme of Ref. [20], but is, instead, a minimal MOM (MiniMOM) scheme, which is defined as follows:

As in every MOM scheme, the gluon and ghost renormalisation constants $Z_{3}$ and $\widetilde{Z}_{3}$ are defined by requiring

$$
\left.Z\left(p^{2}\right)\right|_{p^{2}=\mu^{2}}=1 \text { and }\left.G\left(p^{2}\right)\right|_{p^{2}=\mu^{2}}=1,
$$

\footnotetext{
${ }^{2}$ In order to do that one needs a proper non-perturbative definition of BRST symmetry on the lattice which is possible in principle, but not realised in present lattice implementations of Landau gauge. For a recent discussion, see [14].
}

the perturbative realisation of (2) valid for $\mu^{2} \gg \Lambda^{2}$ where $\Lambda$ is the scale parameter of the scheme. $Z\left(p^{2}\right)$ and $G\left(p^{2}\right)$ are the dressing functions in the renormalised gluon and ghost propagators of Landau-gauge QCD, which are in (Euclidean) momentum space of the form

$$
D_{\mu \nu}^{a b}(p)=\delta^{a b}\left(\delta^{\mu \nu}-\frac{p_{\mu} p_{v}}{p^{2}}\right) \frac{Z\left(p^{2}\right)}{p^{2}}
$$

and

$$
D_{G}^{a b}(p)=-\delta^{a b} \frac{G\left(p^{2}\right)}{p^{2}},
$$

respectively. Instead of imposing additional analogous renormalisation conditions on vertex functions, requiring certain vertex structures to equal their tree-level counter parts at some symmetric or asymmetric subtraction point, we here supplement (4) by the further condition

$$
\widetilde{Z}_{1}=\widetilde{Z}_{1}^{\overline{\mathrm{MS}}}
$$

where $\widetilde{Z}_{1}$ is the renormalisation constant of the ghost-gluon vertex, whose momentum dependence is thus the same as in the minimal subtraction schemes. The renormalisation constants for the three and four gluon vertex functions are then determined by the Slavnov-Taylor identities as usual,

$$
Z_{1}=\frac{Z_{3}}{\widetilde{Z}_{3}} \widetilde{Z}_{1} \text { and } Z_{4}=\frac{Z_{3}}{\widetilde{Z}_{3}^{2}} \widetilde{Z}_{1}^{2} .
$$

The extension to quarks is straightforward, and this defines the MiniMOM scheme: momentum subtraction for the gluon, ghost and quark propagators, and minimal ( $\overline{\mathrm{MS}})$ subtraction for the ghost-gluon vertex (7) together with the Slavnov-Taylor identities to fix the remaining vertices, including the quarkgluon vertex. Note that the renormalisation constants for these remaining vertices then differ from those in the $\overline{\mathrm{MS}}$ scheme by ratios of the propagator (field) renormalisation constants in the MiniMOM (MM) and $\overline{\mathrm{MS}}$ schemes, for example,

$$
Z_{1}^{\mathrm{MM}}=\frac{Z_{3}^{\mathrm{MM}}}{Z_{3}^{\overline{\mathrm{MS}}}} \frac{\widetilde{Z_{3}^{\mathrm{MS}}}}{\widetilde{Z}_{3}^{\mathrm{MM}}} Z_{1}^{\overline{\mathrm{MS}}} \quad \text { and } \quad Z_{4}^{\mathrm{MM}}=\frac{Z_{3}^{\mathrm{MM}}}{Z_{3}^{\overline{\mathrm{MS}}}}\left(\frac{\widetilde{Z}_{3}^{\overline{\mathrm{MS}}}}{\widetilde{Z}_{3}^{\mathrm{MM}}}\right)^{2} Z_{4}^{\overline{\mathrm{MS}}}
$$

Likewise, from the general definition of the renormalised coupling constant,

$$
\alpha_{s}(\mu)=\frac{g^{2}(\mu)}{4 \pi}=\frac{Z_{3} \widetilde{Z}_{3}^{2}}{\widetilde{Z}_{1}^{2}} \frac{g_{\text {bare }}^{2}}{4 \pi},
$$

the MiniMOM and $\overline{\mathrm{MS}}$ scheme couplings, with the definition $\widetilde{Z}_{1}^{\mathrm{MM}} \equiv \widetilde{Z}_{1}^{\overline{\mathrm{MS}}}$ in $(7)$, are related by

$$
\alpha_{s}^{\mathrm{MM}}(\mu)=\frac{Z_{3}^{\mathrm{MM}}}{Z_{3}^{\overline{\mathrm{MS}}}}\left(\frac{\widetilde{Z}_{3}^{\mathrm{MM}}}{\widetilde{Z}_{3}^{\mathrm{MS}}}\right)^{2} \alpha_{s}^{\overline{\mathrm{MS}}}(\mu)
$$

Note that for this conversion we only need to know the perturbative expansions of the ghost and gluon propagators but not of 
any vertex structures. Rather, with the MOM renormalisation conditions for these propagators (4), or their non-perturbative extension (2) for that matter, we simply obtain,

$$
\frac{\alpha_{s}^{\mathrm{MM}}(\mu)}{\alpha_{s}^{\overline{\mathrm{MS}}}(\mu)}=Z\left(\mu^{2}\right)^{\overline{\mathrm{MS}}} G^{2}\left(\mu^{2}\right)^{\overline{\mathrm{MS}}},
$$

from the gluon and ghost dressing functions evaluated at $p^{2}=$ $\mu^{2}$ in the $\overline{\mathrm{MS}}$ scheme. Alternatively, we can relate the MiniMOM coupling to the MOMh scheme just as easily via

$$
\alpha_{s}^{\mathrm{MM}}(\mu)=\left(\frac{\widetilde{Z}_{1}^{\mathrm{MOMh}}}{\widetilde{Z_{1}^{\mathrm{MS}}}}\right)^{2} \alpha_{s}^{\mathrm{MOMh}}(\mu) .
$$

All these conversion identities are valid for arbitrary linearcovariant gauges and not restricted to Landau gauge. The special feature of Landau gauge is that there $\widetilde{Z}_{1}^{\overline{\mathrm{MS}}}=\widetilde{Z}_{1}^{\mathrm{MM}}=1$. Because the Landau gauge ghost-gluon vertex trivially reduces to its tree-level form when one of the ghost momenta is set to zero, the MiniMOM scheme then agrees with the asymmetric $\widetilde{\mathrm{MOM}}$ scheme of Ref. [21] (called the $T$ scheme in Ref. [19]) which is defined from renormalising precisely this vertex structure. The MiniMOM scheme is defined, however, so as to not require knowledge of any vertex structure beyond the $\overline{\mathrm{MS}}$ scheme contributions as determined by their ultra-violet divergences. This makes it particularly useful for a lattice determination of $\alpha_{s}$ from the perturbative behaviour of QCD Green's functions as described in Refs. [5, 6, 7].

For early 2 and 3-loop calculations in Feynman gauge, see [23]. The complete 2-loop results for general gauge parameters are given in [24]. To obtain the 4-loop version of the conversion between $\alpha_{s}^{\overline{\mathrm{MS}}}$ and $\alpha_{s}^{\mathrm{MM}}$ from Eq. (12), we use the 3-loop expressions for the gluon and ghost self-energies found in Appendix $\mathrm{C}$ of [21] which, with $a \equiv \alpha_{s}^{\overline{\mathrm{MS}}} / \pi$, for $N_{f}$ massless quarks yields

$$
\alpha_{s}^{\mathrm{MM}} / \alpha_{s}^{\overline{\mathrm{MS}}}=1+D_{1} a+D_{2} a^{2}+D_{3} a^{3}+O\left(a^{4}\right),
$$

where, with $\zeta_{3}=1.2020569, \zeta_{5}=1.0369278$, and $C_{F}=\left(N_{c}^{2}-\right.$ 1) $/ 2 N_{c}$,

$$
\begin{aligned}
& D_{1}=d_{10}+d_{11} N_{f}, \\
& d_{10}=\left[\frac{169}{144}+\frac{1}{8} \xi+\frac{1}{16} \xi^{2}\right] N_{c}, \quad d_{11}=-\frac{5}{18} . \\
& D_{2}=d_{20}+d_{21} N_{f}+d_{22} N_{f}^{2}, \\
& d_{20}=\left[\frac{76063}{20736}-\frac{39}{128} \zeta_{3}+\left(\frac{269}{2304}+\frac{11}{64} \zeta_{3}\right) \xi+\right. \\
&\left.\left(\frac{35}{256}-\frac{3}{128} \zeta_{3}\right) \xi^{2}+\frac{5}{256} \xi^{3}\right] N_{c}^{2}, \\
& d_{21}=-\left[\frac{55}{96}-\frac{1}{2} \zeta_{3}\right] C_{f}-\left[\frac{1583}{1296}+\frac{1}{4} \zeta_{3}+\frac{5}{144} \xi\right] N_{c}, \\
& d_{22}= \frac{25}{324} \cdot \\
& D_{3}=d_{30}+d_{31} N_{f}+d_{32} N_{f}^{2}+d_{33} N_{f}^{3}, \\
& d_{30=} {\left[\frac{42074947}{2985984}-\frac{20225}{9216} \zeta_{3}-\frac{7805}{12288} \zeta_{5}+\right.} \\
&\left(\frac{17743}{41472}+\frac{10335}{9216} \zeta_{3}-\frac{295}{1024} \zeta_{5}\right) \xi+ \\
&\left(\frac{16235}{36864}-\frac{71}{1536} \zeta_{3}-\frac{175}{6144} \zeta_{5}\right) \xi^{2}+
\end{aligned}
$$

Table 1: The numerical values of the $D_{k}$ in the 4-loop relation between $\alpha_{s}^{\mathrm{MM}}$ and $\alpha_{s}^{\overline{\mathrm{MS}}}$ in Landau gauge, Eqs. 14 and 15 with $\xi=0$, for $N_{c}=3$ and $N_{f}=0,2$, and 3 .

\begin{tabular}{rrrr}
\hline & $N_{f}=0$ & $N_{f}=2$ & $N_{f}=3$ \\
\hline$D_{1}$ & 3.52083333 & 2.96527778 & 2.6875 \\
$D_{2}$ & 29.71718945 & 20.96900712 & 16.82639745 \\
$D_{3}$ & 291.4436449 & 175.9308786 & 127.6686773 \\
\hline
\end{tabular}

$$
\begin{aligned}
& \left(\frac{1207}{12288}-\frac{61}{3072} \zeta_{3}-\frac{5}{3072} \zeta_{5}\right) \xi^{3}+ \\
& \left.\left(\frac{169}{12288}-\frac{11}{3072} \zeta_{3}+\frac{35}{12288} \zeta_{5}\right) \xi^{4}\right] N_{c}^{3}, \\
d_{31}= & {\left[-\frac{202997}{31104}-\frac{217}{288} \zeta_{3}+\frac{5}{12} \zeta_{5}-\right.} \\
& \left.\left(\frac{505}{5184}+\frac{17}{48} \zeta_{3}\right) \xi-\left(\frac{497}{9216}-\frac{1}{128} \zeta_{3}\right) \xi^{2}\right] N_{c}^{2}- \\
& {\left[\frac{41243}{10368}-\frac{41}{16} \zeta_{3}-\frac{5}{8} \zeta_{5}+\left(\frac{55}{768}-\frac{1}{16} \zeta_{3}\right) \xi\right] C_{f} N_{c}+} \\
& {\left[\frac{143}{576}+\frac{37}{48} \zeta_{3}-\frac{5}{4} \zeta_{5}\right] C_{f}^{2}, } \\
d_{32}= & {\left[\frac{47965}{62208}+\frac{37}{144} \zeta_{3}+\left(\frac{7}{2592}+\frac{1}{36} \zeta_{3}\right) \xi\right] N_{c}+} \\
& {\left[\frac{7001}{10368}-\frac{13}{24} \zeta_{3}\right] C_{f}, d_{33}=-\frac{125}{5832} . }
\end{aligned}
$$

This conversion depends on the gauge parameter $\xi \equiv \xi^{\overline{\mathrm{MS}}}$ as in every other momentum subtraction scheme, though in the MiniMOM scheme, this dependence is comparatively weak. At oneloop level, for example, the coefficient of the leading $\xi$ dependence around $\xi=0$ in the above conversion is 3 times smaller than that of the asymmetric $\widehat{\mathrm{MOM}}$ scheme (which coincides with the MiniMOM scheme at $\xi=0$ ).

For $\xi=0$ the same conversion can be obtained from the product of the scheme-invariant propagators given for Landau gauge in Sec. 4 of [25]. We verified the general result [15] for $N_{c}=3$ and $N_{f}=0,3$ and 6 from the explicit expressions given there. Another check of our conversion, up to and including $O\left(a^{2}\right)$, can be obtained using Eq. (13) in Landau gauge, where $\widetilde{Z}_{1}^{\overline{\mathrm{MS}}}=1$, with the 2-loop expression for $\widetilde{Z}_{1}^{\mathrm{MOMh}}(\mu)=\widetilde{\Gamma}^{-1}\left(\mu^{2}\right)$ in the symmetric MOMh scheme from [20], together with the 3-loop conversion from $\alpha_{s}^{\overline{\mathrm{MS}}}$ to $\alpha_{s}^{\mathrm{MOMh}}$ as given there 3

The numerical values of the $D_{k}$ in Landau gauge are given explicitly, for $N_{c}=3$ and $N_{f}=0,2$, and 3, in Tab. 11 As an illustration of the weakness of the dependence on $\xi$, the corresponding $N_{c}=3, N_{f}=3$ results are

$$
\begin{aligned}
& D_{1}=2.6875+0.3750 \xi+0.1875 \xi^{2} \\
& D_{2}=16.8264+2.5977 \xi+0.9769 \xi^{2}+0.1758 \xi^{3} \\
& D_{3}=127.6687+26.7739 \xi+8.3907 \xi^{2}+1.9621 \xi^{3}+0.3349 \xi^{4}
\end{aligned}
$$

with similar, slightly less $\xi$-dependent, results for $N_{f}=0,2$.

In order to compare actual values of $\alpha_{s}$ in the MiniMOM scheme (at $\xi=0$ ) to the corresponding ones in the $\overline{\mathrm{MS}}$ scheme we give two important examples. First, at the mass of the $Z$ boson, $m_{Z}=91.2 \mathrm{GeV}$ with $\alpha_{s}^{\overline{\mathrm{MS}}}\left(m_{Z}^{2}\right)=0.1189$ [1], from Eq. (14]

\footnotetext{
${ }^{3}$ There appears to be a typo in Table 2 of [20], the entry for the 2-loop coefficient of $\widetilde{\Gamma}$ proportional to $N_{c} N_{f}$ should probably read $-0.115(2)$ instead of $-0.151(2)$.
} 
we obtain

$$
\alpha_{s}^{\mathrm{MM}}\left(m_{Z}^{2}\right)=1.096 \alpha_{s}^{\overline{\mathrm{MS}}}\left(m_{Z}^{2}\right),
$$

for $N_{f}=5$, while at the mass of the $\tau$ lepton, $m_{\tau}=1.777 \mathrm{GeV}$,

$$
\alpha_{s}^{\mathrm{MM}}\left(m_{\tau}^{2}\right)=1.59 \alpha_{s}^{\overline{\mathrm{MS}}}\left(m_{\tau}^{2}\right),
$$

where, to be specific, we have used $\alpha_{s}^{\overline{\mathrm{MS}}}\left(m_{\tau}^{2}\right)=0.322$, the value obtained by running $\alpha_{s}^{\overline{\mathrm{MS}}}\left(m_{Z}^{2}\right)=0.1189$ down to the $N_{f}=3$ scale $m_{\tau}^{2}$ using the standard 4-loop running [26].

We conclude this section with a few comments on quark mass effects. As in every off-shell subtraction scheme, the running coupling and beta function of the MiniMOM scheme in principle depend on the quark masses. This is evident from its relation to the (mass independent) $\overline{\mathrm{MS}}$ scheme, Eq. (12), in which the $\overline{\mathrm{MS}}$ scheme gluon and ghost dressing functions will depend on the masses in the quark loops. These have not been included and our conversion formulas are therefore strictly speaking valid only for $N_{f}$ massless quarks. To fully account for finite quark masses at this level one would need the corresponding 3-loop expressions for the gluon and ghost dressing functions with massive quark loops which have not been calculated to our knowledge as yet.

We can estimate the leading quark mass effects, however, which will affect the conversion formula at the 2-loop level. These are obtained from the 1-loop vacuum polarisation with massive fermions in the gluon self-energy, and they lead to an increase of $D_{1}$ (via $d_{11}$ ) as compared to the massless case. For each quark flavour with mass $m_{q}$ one then separately obtains a transcendental function $d_{11}(y)$ of $y \equiv m_{q}^{2} / \mu^{2}$ which approaches $-5 / 18$ for $y \rightarrow 0$, i.e., for $\mu^{2} \gg m_{q}^{2}$. Using the current upper limits from the Particle Data Group for the average up/down mass of $5 \mathrm{MeV}$ and the strange mass of $130 \mathrm{MeV}$ as commonly given at $\mu=2 \mathrm{GeV}$, we observe a maximum increase in $D_{1}$ by $0.15 \%$ at $\mu=2 \mathrm{GeV}$ as compared to the massless $N_{f}=3$ value given in Tab. 11. At $\mu=1 \mathrm{GeV}$, with correspondingly larger light quark masses, the same upper bound for the increase in $D_{1}$ reaches $1 \%$. At larger scales the effect rapidly decreases. In particular, the explicit comparisons in Eqs. (17) and (18) remain unaffected by the corresponding changes in $D_{1}$ (note that even with the charm and bottom quark masses included, the increase in $D_{1}$ will be less than $0.1 \%$ at $\mu=m_{Z}$ as compared to the $N_{f}=5$ massless flavour value there).

The most noticeable quark mass effects will of course occur right at the decoupling scales $\mu=m_{q}$. At the charm threshold, for example, with $\mu=m_{c}=1.27 \mathrm{GeV}$, the quark mass contributions to the vacuum polarisation lead to an increase in $D_{1}$ by around $13 \%$ as compared to the massless $N_{f}=4$ value (at $\mu=2$ $\mathrm{GeV}$ this increase is reduced by a factor of 2 already). Considerable charm-quark mass effects should therefore be expected when converting the 4 flavour MiniMOM coupling to the $\overline{\mathrm{MS}}$ coupling in the phenomenoligically interesting range between 1 and $4 \mathrm{GeV}$. Without a more detailed knowledge of these effects an $N_{f}=4$ conversion would therefore not be advisable. Here we use the MiniMOM to $\overline{\mathrm{MS}}$ conversion up to at most $N_{f}=3$ for which the quark mass effects are very small. For now, matching to the $N_{f}=4$ and 5 regimes should always be done after the conversion, for the $\overline{\mathrm{MS}}$ coupling in the usual way.
There are no charm and bottom quarks in the lattice determinations of the MiniMOM coupling which are presently restricted to $N_{f}=0$ and 2 and which will be extended to $2+1$ light flavours in due course. At the relevant high momentum scales quark mass effects should then be completely negligible in the conversion to $\alpha \overline{\mathrm{MS}}$. In addition, it is always a possibility to remove any small residual light-quark mass effects by extrapolation, if necessary.

\section{Beta-function coefficients of the MiniMOM coupling}

The running of the coupling constant as the scale, $\mu$, changes is controlled by the (scheme-dependent) $\beta$ function which, at small couplings, is defined by

$$
\mu^{2} \frac{d a\left(\mu^{2}\right)}{d \mu^{2}}=\beta(a):=-\sum_{i=0} \beta_{i} a^{i+2},
$$

where $a \equiv \alpha_{s} / \pi$. The $\beta$ function in the $\overline{\mathrm{MS}}$ scheme is known to 4-loop order, the expressions for the corresponding coefficients $\beta_{k}^{\overline{\mathrm{MS}}}, k=0, \cdots, 3$, for general $N_{c}$ and $N_{f}$, being given in Refs. [27]. These results, together with the relation between the $\overline{\mathrm{MS}}$ and MiniMOM coupling given in Eqs. (14) and (15), and the 3-loop version of the expression $\mu^{2} d \xi / d \mu^{2}=\xi \gamma_{3}$, for the running of the renormalised $\overline{\mathrm{MS}}$ gauge parameter, $\xi$, with $\gamma_{3}$ the gluon anomalous dimension, the 3-loop expression for which can be found in Appendix D of Ref. [21], allow us to obtain the $\beta$ function coefficients in the MiniMOM scheme to 4-loop order. For general $N_{c}$ and $N_{f}$, we find

$$
\begin{aligned}
& \beta_{0}^{\mathrm{MM}}= \frac{1}{4}\left[\frac{11}{3} N_{c}-\frac{2}{3} N_{f}\right] \\
& \beta_{1}^{\mathrm{MM}}= \frac{1}{8}\left[\frac{17}{3} N_{c}^{2}-\frac{5}{3} N_{f} N_{c}-C_{f} N_{f}\right]+B_{10}^{\xi}+B_{11}^{\xi} N_{f} \\
& \beta_{2}^{\mathrm{MM}}= {\left[\frac{9655}{4608}-\frac{143}{512} \zeta_{3}\right] N_{c}^{3}-\left[\frac{2009}{2304}+\frac{137}{768} \zeta_{3}\right] N_{f} N_{c}^{2} } \\
&+\left[\frac{23}{384}+\frac{1}{24} \zeta_{3}\right] N_{f}^{2} N_{c}-\left[\frac{641}{1152}-\frac{11}{24} \zeta_{3}\right] C_{f} N_{f} N_{c} \\
&+\frac{1}{64} C_{f}^{2} N_{f}+\left[\frac{23}{288}-\frac{1}{12} \zeta_{3}\right] C_{f} N_{f}^{2} \\
&+B_{20}^{\xi}+B_{21}^{\xi} N_{f}+B_{22}^{\xi} N_{f}^{2}, \\
& \beta_{3}^{\mathrm{MM}}=\left.\frac{1381429}{165888}-\frac{225335}{110592} \zeta_{3}-\frac{85855}{73728} \zeta_{5}\right] N_{c}^{4} \\
&+\left[-\frac{244549}{55296}+\frac{3395}{18432} \zeta_{3}+\frac{35965}{3686} \zeta_{5}\right] N_{f} N_{c}^{3} \\
&+\left[-\frac{5}{96}+\frac{11}{8} \zeta_{3}-\left(\frac{60685}{18432}-\frac{85}{64} \zeta_{3}-\frac{55}{48} \zeta_{5}\right) C_{f} N_{f}\right. \\
&\left.\quad+\left(\frac{14807}{27648}+\frac{125}{768} \zeta_{3}-\frac{5}{36} \zeta_{5}\right) N_{f}^{2}\right] N_{c}^{2} \\
&+\left[\left(\frac{1}{36}-\frac{13}{48} \zeta_{3}\right) N_{f}+\left(\frac{527}{4608}+\frac{143}{96} \zeta_{3}-\frac{55}{24} \zeta_{5}\right) C_{f}^{2} N_{f}\right. \\
&+\left(\frac{2357}{2304}-\frac{43}{96} \zeta_{3}-\frac{5}{24} \zeta_{5}\right) C_{f} N_{f}^{2} \\
&\left.-\left(\frac{7}{648}+\frac{7}{432} \zeta_{3}\right) N_{f}^{3}\right] N_{c} \\
&+\frac{23}{256} C_{f}^{3} N_{f}+\left(\frac{11}{576}-\frac{1}{24} \zeta_{3}\right) N_{f}^{2} \\
&-\left(\frac{29}{1152}+\frac{1}{3} \zeta_{3}-\frac{5}{12} \zeta_{5}\right) C_{f}^{2} N_{f}^{2} \\
&\left.+B_{31}^{\xi} N_{3}\right) C_{f} N_{f}^{3}-\frac{11}{192} \frac{N_{f}^{2}}{N_{c}^{2}}+\frac{1}{8} \zeta_{3} \frac{N_{f}^{2}}{N_{c}^{2}} \\
& N_{f}^{2}+B_{33}^{\xi} N_{f}^{3} \\
& \\
&
\end{aligned}
$$


Table 2: The $B^{\xi}$ terms in Eqs. (20) for $N_{c}=3$.

$$
\begin{aligned}
B_{10}^{\xi}= & -\frac{39}{64} \xi-\frac{15}{32} \xi^{2}+\frac{9}{64} \xi^{3}, \quad B_{11}^{\xi}=\frac{1}{16} \xi+\frac{1}{16} \xi^{2} \\
B_{20}^{\xi}= & \left.-\frac{7351}{2048}+\frac{891}{512} \zeta_{3}\right] \xi+\left[\frac{2177}{2048}+\frac{351}{512} \zeta_{3}\right] \xi^{2} \\
& -\left[\frac{225}{2048}+\frac{81}{512} \zeta_{3}\right] \xi^{3}+\frac{531}{2048} \xi^{4}-\frac{81}{1024} \xi^{5} \\
B_{21}^{\xi}= & \frac{101}{384} \xi-\left[\frac{59}{1536}+\frac{9}{256} \zeta_{3}\right] \xi^{2}+\frac{3}{32} \xi^{3}-\frac{15}{512} \xi^{4} \\
B_{22}^{\xi}= & \frac{5}{288} \xi+\frac{5}{144} \xi^{2}, \quad B_{33}^{\xi}=\left[-\frac{43}{5184}+\frac{1}{72} \zeta_{3}\right] \xi+\frac{25}{1728} \xi^{2} \\
B_{30}^{\xi}= & \left.-\frac{3791075}{98304}+\frac{140271}{2048} \zeta_{3}-\frac{246915}{8192} \zeta_{5}\right] \xi \\
& -\left[\frac{459983}{32768}-\frac{31887}{4096} \zeta_{3}+\frac{19035}{4096} \zeta_{5}\right] \xi^{2} \\
& -\left[\frac{24561}{16384}-\frac{3681}{8192} \zeta_{3}+\frac{2475}{4096} \zeta_{5}\right] \xi^{3} \\
& +\left[\frac{11277}{8192}-\frac{6327}{4096} \zeta_{3}+\frac{7155}{8192} \zeta_{5}\right] \xi^{4} \\
& +\left[\frac{2727}{4096}+\frac{513}{8192} \zeta_{3}-\frac{945}{8192} \zeta_{5}\right] \xi^{5}-\frac{2619}{16384} \xi^{6}+\frac{729}{16384} \xi^{7} \\
B_{31}^{\xi}= & \left.\frac{203483}{24576}-\frac{19821}{2048} \zeta_{3} \frac{2655}{2048} \zeta_{5}\right] \xi+\left[\frac{38057}{12288}-\frac{345}{512} \zeta_{3}\right] \xi^{2} \\
& +\left[\frac{1569}{2048}-\frac{39}{128} \zeta_{3}-\frac{15}{2048} \zeta_{5}\right] \xi^{3} \\
& +\left[\frac{81}{256}+\frac{21}{1024} \zeta_{3}-\frac{315}{4096} \zeta_{5}\right] \xi^{4}-\frac{513}{4096} \xi^{5}+\frac{63}{4096} \xi^{6} \\
B_{32}^{\xi}= & \left.-\frac{11}{36}+\frac{251}{384} \zeta_{3}\right] \xi-\left[\frac{119}{3072}-\frac{3}{256} \zeta_{3}\right] \xi^{2}+\frac{15}{512} \xi^{3}-\frac{5}{128} \xi^{4} \\
\hline &
\end{aligned}
$$

where the $B_{m n}^{\xi}$ all vanish in Landau gauge 4 The general expressions for the $B_{m n}^{\xi}$ are rather long and unilluminating, and hence not included here. The results for the phenomenologically most interesting case, $N_{c}=3$, however, are given in Tab. 2.

The numerical values of the $\beta_{i}^{\mathrm{MM}}$ 's in Landau gauge are given, for $N_{c}=3$ and $N_{f}=0,2$ and 3, in Tab. 3] For the reader's convenience, we give also the numerical results for general $\xi$ and $N_{f}=N_{c}=3$,

$$
\begin{aligned}
\beta_{0}^{\mathrm{MM}}= & 2.25 \\
\beta_{1}^{\mathrm{MM}}= & 4.0-0.421875 \xi-0.28125 \xi^{2}+0.140625 \xi^{3} \\
\beta_{2}^{\mathrm{MM}}= & 20.9183-0.552182 \xi-0.168435 \xi^{2} \\
& -0.0187824 \xi^{3}+0.171387 \xi^{4}-0.0791016 \xi^{5} \\
\beta_{3}^{\mathrm{MM}}= & 160.771+10.5774 \xi-2.46840 \xi^{2} \\
& -0.145040 \xi^{3}+0.857841 \xi^{4}+0.245698 \xi^{5} \\
& -0.113708 \xi^{6}+0.0444946 \xi^{7}
\end{aligned}
$$

which results serve to illustrate the weakness of the $\xi$ dependence in the vicinity of Landau gauge. Note that, while the first coefficient, $\beta_{0}^{\mathrm{MM}}$ is gauge independent, and universal, the coefficients beginning with $\beta_{1}^{\mathrm{MM}}$ are gauge dependent, as is typical of momentum subtraction schemes (as usual, the universal value of $\beta_{1}^{\mathrm{MM}}$ is obtained only in Landau gauge).

\section{Comparing perturbative expansions}

The definition of the running coupling in (1) has been widely used, and continues to be widely used, in phenomenological

\footnotetext{
${ }^{4}$ The $N_{c}=3$ Landau gauge version of these results were first presented in Refs. [6, 7] and subsequently confirmed in Ref. [19].
}

Table 3: The $\beta_{i}^{\mathrm{MM}}$ in Landau gauge $(\xi \equiv 0)$ for different $N_{f}$ and $N_{c}=3$.

\begin{tabular}{lrrr}
\hline & $N_{f}=0$ & $N_{f}=2$ & $N_{f}=3$ \\
\hline$\beta_{0}^{\mathrm{MM}}$ & 2.75 & $2.41 \overline{66}$ & 2.25 \\
$\beta_{1}^{\mathrm{MM}}$ & 6.375 & $4.791 \overline{66}$ & 4.00 \\
$\beta_{2}^{\mathrm{MM}}$ & 47.5075357 & 29.1756592 & 20.9183135 \\
$\beta_{3}^{\mathrm{MM}}$ & 392.7385102 & 226.4690053 & 160.7710385 \\
\hline
\end{tabular}

applications of QCD Green's functions within non-perturbative continuum approaches [12, 22] based on DSEs or FRGEs, for example. The precise definition of the underlying renormalisation scheme, the MiniMOM scheme, puts these approaches on a firmer ground, and should serve to resolve any previous misunderstandings. We have already stressed its utility in providing a route to a lattice determination of $\alpha_{s}$ requiring only a calculation of two-point functions, which are relative easy to determine with high precision in current simulations. Here we show, as an added bonus, that the MiniMOM coupling may provide a useful alternative to the $\overline{\mathrm{MS}}$ coupling in certain phenomenological applications. We do so by considering the expressions for the perturbative contribution to quantities relevant to a selection of phenomenological applications in the $N_{f}=3$ regime, expanded in terms of either the $\overline{\mathrm{MS}}$ or the MiniMOM coupling. With $a_{\overline{\mathrm{MS}}}=\alpha_{s}^{\overline{\mathrm{MS}}}\left(Q^{2}\right) / \pi, a_{\mathrm{MM}}=\alpha_{s}^{\mathrm{MM}}\left(Q^{2}\right) / \pi, D_{1}, D_{2}$ and $D_{3}$ from Eqs. (15), and

$$
C_{1}=-D_{1}, \quad C_{2}=-D_{2}+2 D_{1}^{2} \text { and } C_{3}=-D_{3}+5 D_{1} D_{2}-5 D_{1}^{3}
$$

an observable, $O$, whose $\overline{\mathrm{MS}}$ expansion is

$$
O=1+A_{1} a_{\overline{\mathrm{MS}}}+A_{2} a_{\overline{\mathrm{MS}}}^{2}+A_{3} a_{\overline{\mathrm{MS}}}^{3}+\ldots,
$$

has an equivalent MiniMOM coupling expansion

$$
\begin{aligned}
O=1 & +A_{1} a_{\mathrm{MM}}+\left[A_{2}+C_{1} A_{1}\right] a_{\mathrm{MM}}^{2} \\
& +\left[A_{3}+2 C_{1} A_{2}+C_{2} A_{1}\right] a_{\mathrm{MM}}^{3} \\
& +\left[A_{4}+3 C_{1} A_{3}+\left(2 C_{2}+C_{1}^{2}\right) A_{2}+C_{3} A_{1}\right] a_{\mathrm{MM}}^{4}+\ldots
\end{aligned}
$$

In investigating the phenomenological utility of the MiniMOM coupling, we will consider the $N_{c}=N_{f}=3$ case, for which $C_{1}=-2.6875, C_{2}=-2.38108495$ and $C_{3}=1.3815951$. We show that, in some of the considered cases, use of the MiniMOM coupling significantly improves the apparent convergence of the relevant perturbative series, while, in other cases, it does not. Whether or not it is useful to employ the MiniMOM coupling is thus something to be decided on a case to case basis.

\subsection{The Adler function of the vector/axial vector current cor- relators}

Our first example is the dimension $D=0$ contribution to the Adler function, $D_{V / A ; i j}\left(Q^{2}\right)=-Q^{2} d \Pi_{V / A ; i j}\left(Q^{2}\right) / d Q^{2}$, of the flavour $i j$ vector $(\mathrm{V})$ or axial vector (A) current scalar correlator, $\Pi_{V / A ; i j}$. At scales of phenomenological interest, $\left.D_{V / A ; i j}\left(Q^{2}\right)\right|_{D=0}$ is far and away the dominant term on the OPE 
side of finite energy sum rules (FESRs) which have been studied in the literature based on either electroproduction crosssections or hadronic $\tau$ decay data. The $\tau$-based FESRs are used in precision determinations of $\alpha_{s}\left(M_{Z}\right)$, the most recent of which are described in Refs. [28, 29, 30]. A combination of electroproduction- and $\tau$-based FESRs has also been used to investigate the present discrepancy [31, 32] between the electroproduction and $\tau$ version of the $I=1 \mathrm{~V}$ spectral function [33], a discrepancy which prevents a clear decision as to whether or not the Standard Model (SM) prediction for $(g-2)_{\mu}$ is compatible with the current high-precision experimental result [34].

$\left.D_{V / A ; i j}\right|_{D=0}$ is known to 5-loops [35] and, for $N_{c}=N_{f}=3$, given in terms of $a_{\overline{\mathrm{MS}}}$, by

$$
\begin{aligned}
\left.4 \pi^{2} D_{V / A ; i j}\right|_{D=0}=1 & +a_{\overline{\mathrm{MS}}}+1.6398 a_{\overline{\mathrm{MS}}}^{2} \\
& +6.3710 a_{\overline{\mathrm{MS}}}^{3}+49.0757 a_{\overline{\mathrm{MS}}}^{4}+\ldots .
\end{aligned}
$$

The equivalent expansion in terms of $a_{\mathrm{MM}}$ is

$$
\begin{aligned}
\left.4 \pi^{2} D_{V / A ; i j}\right|_{D=0}=1 & +a_{\mathrm{MM}}-1.0477 a_{\mathrm{MM}}^{2} \\
& -4.8241 a_{\mathrm{MM}}^{3}+3.1257 a_{\mathrm{MM}}^{4}+\ldots
\end{aligned}
$$

which displays significantly improved convergence at scales relevant to the phenomenological studies noted above $(\sim 2-$ $4 \mathrm{GeV}^{2}$ ), even when one takes into account the increased size of $\alpha_{s}^{\mathrm{MM}}\left(Q^{2}\right)$ as compared to $\alpha_{s}^{\overline{\mathrm{MS}}}\left(Q^{2}\right)$. This improved convergence will also be manifest in FESR studies which employ "contour improved perturbation theory" (CIPT) in their evaluations of the relevant weighted $D=0$ integrals 5

\subsection{The second derivative of the $D=0$ part of the scalar/ pseudoscalar correlator}

As our second example, we consider the subtractionconstant-independent second derivatives, $\Pi_{S / P S ; i j}^{\prime \prime}\left(Q^{2}\right)$, of the $D=0$ part of the scalar (S) and pseudoscalar (PS) correlators, $\Pi_{S ; i j}$ and $\Pi_{P S ; i j}$, formed from the divergences of the flavour $i j$ $\mathrm{V}$ or A currents. These quantities, which are equal for the $\mathrm{S}$ and

${ }^{5}$ The CIPT prescription employs the truncated expansion of Eq. 25 pointby-point along the circle $\left|Q^{2}\right|=s_{0}$ in the complex $Q^{2}$-plane. An alternate approach to evaluating the weighted $D=0$ contour integrals is to use the "fixed order perturbation theory" (FOPT) prescription, in which the series is expanded, and truncated, using the running coupling at the same fixed scale, e.g., $\mu^{2}=s_{0}$, for all points on the circle. In the FOPT scheme, large logs are unavoidable over some portion of the contour. Nonetheless, recent arguments [28], based on a model constrained by known features of the large order behaviour of the $D=0$ perturbative series for $D_{V / A ; i j}\left(Q^{2}\right)$, shows it is possible that the truncated FOPT form might provide a more reliable representation of the resummed series than would the CIPT form. This is potentially relevant here because the improvement in the convergence of the integrated FOPT series achieved through the use of the MiniMOM couplant is, for commonly used weights, far less compelling than that achieved in the CIPT case. While a study of the 5-loop FOPT approximation to a range of weighted integrals of the model for the resummed series in Ref. [28] shows that a good representation of the corresponding data integrals is not possible, in contrast to the situation when the 5-loop CIPT evaluation is employed [36], the analogous study has not yet been performed for the full resummed model, and, as a result, the preference for the CIPT approach (where the improvement due to the re-ordering of the series using $a^{\mathrm{MM}}$ would be operative) is not yet conclusive.
PS cases, apart from the overall $\left(m_{i} \mp m_{j}\right)^{2}$ factors, are the dominant terms on the OPE side of S and PS FESRs and Borel sum rules (BSRs) which provide the most reliable sum rule determinations of $m_{s}$ and $m_{u}+m_{d}$ [37, 38, 39]. Useful lower bounds on $m_{s}$ have also been obtained from the PS $i j=u s$ sum rules using a combination of the accurately known $K$ pole contribution and spectral positivity [39, 40, 41]. A combination of FESRs and BSRs based on $\Pi_{P S ; u s}$, in addition, provides a determination, not just of $m_{s}$, but also of the decay constants of the first two excited $K$ resonances, and hence a useful, highly constrained model of the $u s$ PS spectral function, a model which, combined with the $u s \mathrm{~S}$ spectral function constructed in Refs. [38], allows the continuum $J=0$ spectral contributions to be subtracted from the experimental differential distribution in strange hadronic $\tau$ decays. This turns out to be a crucial input to the hadronic $\tau$ decay determination of $\left|V_{u s}\right|$ [42, 43, 44, 45] since the OPE representation of the $J=0$ contributions is extremely badly behaved at all kinematically accessible scales, preventing one from employing FESRs based on the full experimental differential distribution [46].

The expansion of $\Pi_{S / P S ; i j}^{\prime \prime}$ in terms of $a_{\overline{\mathrm{MS}}}$ is known to five loops [39] and, for $N_{c}=N_{f}=3$, is given by

$$
\begin{aligned}
& \left.\Pi_{S / P S ; i j}^{\prime \prime}\left(Q^{2}\right)\right|_{D=0}=\frac{3\left[\left(m_{i} \mp m_{j}\right)\left(Q^{2}\right)\right]^{2}}{8 \pi^{2} Q^{2}}\left[1+\frac{11}{3} a_{\overline{\mathrm{MS}}}+\right. \\
& \left.+14.17928 a_{\overline{\mathrm{MS}}}^{2}+77.36834 a_{\overline{\mathrm{MS}}}^{3}+511.82848 a_{\overline{\mathrm{MS}}}^{4}+\ldots\right]
\end{aligned}
$$

where $m_{i}\left(Q^{2}\right)$ is the running quark mass in the $\overline{\mathrm{MS}}$ scheme. Reexpressing the series in terms of $a_{\mathrm{MM}}$ yields

$$
\begin{aligned}
& \left.\Pi_{S / P S ; i j}^{\prime \prime}\left(Q^{2}\right)\right|_{D=0}=\frac{3\left[\left(m_{i} \mp m_{j}\right)\left(Q^{2}\right)\right]^{2}}{8 \pi^{2} Q^{2}}\left[1+\frac{11}{3} a_{\mathrm{MM}}+\right. \\
& \left.+4.32512 a_{\mathrm{MM}}^{2}-7.57595 a_{\mathrm{MM}}^{3}-71.99997 a_{\mathrm{MM}}^{4}+\ldots\right],
\end{aligned}
$$

which again displays significantly improved convergence. Such improved convergence is likely to allow a significant reduction in the errors on the determination of the light quark masses, and an improved version of the light quark mass bounds.

\subsection{The leading $D=2$ contribution to the flavour-breaking ud-us, $V+A, J=0+1$ correlator difference}

The flavour-breaking correlator difference, $\Delta \Pi_{\tau} \equiv \Pi_{V+A ; u d}^{(0+1)}-$ $\Pi_{V+A ; u s}^{(0+1)}$, where the superscript $(0+1)$ denotes the sum of $J=$ 0 and 1 contributions, is of interest in extracting $\left|V_{u s}\right|$ (and/or $m_{s}$ ) from hadronic $\tau$ decay data. The leading term in the OPE representation of $\Delta \Pi_{\tau}$ is the $D=2$ mass-dependent perturbative contribution, $\left.\Delta \Pi_{\tau}\right|_{D=2} ^{\mathrm{OPE}}$, proportional to $m_{s}^{2}$. FESRs based on the $J=0+1$ combination are employed because of the very bad behaviour of the OPE representation of the related integrated $D=2, J=0$ contribution [46]. Even after the subtraction of $J=0$ spectral contributions made possible by the $u s \mathrm{~S}$ and PS studies noted above [37, 38], the $\left|V_{u s}\right|$ (and/or $m_{s}$ ) extraction is complicated by the slow convergence of the $D=2, J=0+1$ series at the correlator level. $\left.\Delta \prod_{\tau}\right|_{D=2} ^{\mathrm{OPE}}$ is known to 4-loop order 
and, for $N_{c}=N_{f}=3$, neglecting corrections of $O\left(m_{u}^{2} / m_{s}^{2}\right)$, has the form [47]

$$
\begin{aligned}
\left.\Delta \Pi_{\tau}\left(Q^{2}\right)\right|_{D=2} ^{\mathrm{OPE}}= & \frac{3 m_{s}^{2}\left(Q^{2}\right)}{2 \pi^{2} Q^{2}}\left[1+\frac{7}{3} a_{\overline{\mathrm{MS}}}+19.93313 a_{\overline{\mathrm{MS}}}^{2}\right. \\
& \left.+208.746 a_{\overline{\mathrm{MS}}}^{3}+(2378) a_{\overline{\mathrm{MS}}}^{4}+\ldots\right]
\end{aligned}
$$

where the PMS/FAC estimate for the 5-loop coefficient, 2378 [47], has been included for exploration purposes. Since $a_{\overline{\mathrm{MS}}}\left(m_{\tau}^{2}\right) \sim 0.1$, convergence is rather slow at the space-like point on the FESR contour. It would be very helpful in reducing theoretical uncertainties in the determination of $\left|V_{u s}\right|$ by this method were the use of the alternate couplant, $a_{\mathrm{MM}}$, to significantly improve the convergence of the $D=2, J=0+1$ series. Recasting (28) terms of $a_{\mathrm{MM}}$, we find

$$
\begin{aligned}
& \left.\Delta \Pi_{\tau}\left(Q^{2}\right)\right|_{D=2} ^{\mathrm{OPE}}=\frac{3 m_{s}^{2}\left(Q^{2}\right)}{2 \pi^{2} Q^{2}}\left[1+\frac{7}{3} a_{\mathrm{MM}}+13.66230 a_{\mathrm{MM}}^{2}\right. \\
& \left.\quad+96.04956 a_{\mathrm{MM}}^{3}+(747.25429) a_{\mathrm{MM}}^{4}+\ldots\right]
\end{aligned}
$$

Unfortunately, even at scales $\sim m_{\tau}^{2} \simeq 3.16 \mathrm{GeV}^{2}$, where $a_{\mathrm{MM}}\left(m_{\tau}^{2}\right) \sim 0.15$, the decrease in the coefficient sizes for this alternate representation is roughly compensated for by the increased size of $a_{\mathrm{MM}}$ as compared to $a_{\overline{\mathrm{MS}}}$. Thus, in this case, the MiniMOM coupling does not produce a useful re-ordering of the original series.

\section{Conclusions}

Our main intention here was to provide the precise definition together with a perturbative analysis of the MiniMOM scheme underlying the running coupling in (1) which has been widely used in non-perturbative studies of the infrared behaviour of QCD Green's functions and phenomenological applications since its introduction more than 12 years ago in Refs. [4].

The particular occasion for this probably overdue clarification is the relatively recent and promising effort to determine this coupling from lattice simulations. The high precision and reliable error estimates desirable for this project require the improved perturbative knowledge which our relation between the MiniMOM and the $\overline{\mathrm{MS}}$ couplings at 4-loop order provides. When determining the MiniMOM coupling from the gluon and ghost propagators of lattice Landau gauge in Monte Carlo simulations, with discretisation errors of $O\left(a^{2}\right)$, one has

$$
\alpha_{s}^{\mathrm{MM}}\left(q^{2}\right)=\frac{g^{2}(a)}{4 \pi} Z_{L}\left(q^{2}, a^{2}\right) G_{L}^{2}\left(q^{2}, a^{2}\right)+O\left(a^{2}\right),
$$

as $a \rightarrow 0$, with $g^{2}(a)$ the bare coupling at the lattice cutoff scale $1 / a$, and $Z_{L}$ and $G_{L}$ are the bare lattice gluon and ghost dressing functions, respectively (see [5] for details). It turns out that $\alpha_{s}^{\mathrm{MM}}$ can be determined quite accurately over a wide range of scales. Once the lattice scale is fixed, this translates into a highprecision determination of $\Lambda^{\mathrm{MM}}$. $\Lambda^{\overline{\mathrm{MS}}}$ can then be determined via

$$
\frac{\Lambda^{\mathrm{MM}}}{\Lambda^{\overline{\mathrm{MS}}}}=\exp \left(\frac{D_{1}}{2 \beta_{0}}\right)
$$

Table 4: $\Lambda^{\mathrm{MM}} / \Lambda^{\overline{\mathrm{MS}}}$ in Landau gauge for $N_{c}=3$ and different $N_{f}$.

\begin{tabular}{lllll}
\hline$N_{f}=0$ & $N_{f}=2$ & $N_{f}=3$ & $N_{f}=4$ & $N_{f}=5$ \\
\hline 1.8968 & 1.8469 & 1.8171 & 1.7831 & 1.7440 \\
\hline
\end{tabular}

where $D_{1}$ is given by Eq. (15a) and, with our normalisation, for $N_{c}=N_{f}=3$ for example, $\beta_{0}=9 / 4$. Numerical values for the $N_{c}=3$, Landau gauge version of this conversion factor are given in Tab. 4 A thorough up-to-date analysis using the available lattice data for $N_{f}=0,2$ is given elsewhere [8].

Finally, as we have demonstrated in the previous section, it turns out that the MiniMOM coupling is also well suited for use in perturbative analyses in the place of the commonly used $\overline{\mathrm{MS}}$ coupling, since the relation between the two is known to 4-loop order. We have compared the corresponding perturbative expansions of a selection of observables of phenomenological interest and found that the MiniMOM coupling appears to provide an efficient re-ordering of the leading $D=0$ perturbative contributions for the V/A and S/PS correlator cases. One should bear in mind, however, that this improvement is not universal, as the example of the $D=2$ contribution to the flavour-breaking, $J=0+1, V+A$ correlator difference shows, though this case does not create a dramatic problem either. Those examples where significant improvement is found, however, suggest it may be useful to consider $\alpha_{s}^{\mathrm{MM}}$ as an alternative expansion parameter for the perturbative series relevant to other observables, such as those relevant to the decays of heavy quarkonia and heavy quark physics, as well.

\section{Acknowledgements}

This research was supported by the Australian Research Council. K. M. acknowledges the ongoing support of the Natural Sciences and Engineering Council of Canada, and the hospitality of the CSSM at the University of Adelaide.

\section{References}

[1] S. Bethke, Prog. Part. Nucl. Phys. 58 (2007) 351 arXiv:hep-ex/0606035].

[2] K. Maltman, D. Leinweber, P. Moran, A. Sternbeck, Phys. Rev. D78 (2008) 114504 arXiv:0807.2020].

[3] C. T. H. Davies, et al., Phys. Rev. D78 (2008) 114507 arXiv:0807.1687.

[4] L. von Smekal, R. Alkofer, A. Hauck, Phys. Rev. Lett. 79 (1997) 3591 [arXiv: hep-ph/9705242]; L. von Smekal, A. Hauck, R. Alkofer, Ann. Phys. 267 (1998) 1 |arXiv:hep-ph/9707327|.

[5] A. Sternbeck, et al., PoS LAT2007 (2007) 256 |arXiv:0710.2965|.

[6] L. von Smekal, Infrared exponents and the strong coupling limit of Lattice Landau gauge, Talk at Quarks and Hadrons in strong QCD, St. Goar, Germany, http://crunch.ikp.physik.tu-darmstadt.de/qhqcd (March 2008).

[7] A. Sternbeck, Four-loop $\alpha_{s}$ in a minimal MOM scheme, Talk at T(r)opical QCD, Port Douglas, Australia (August 2008).

[8] A. Sternbeck et al., presented at the XXVII th International Symposium on Lattice Field Theory, Lattice 2009, Beijing, July 2009.

[9] C. Lerche, L. von Smekal, Phys. Rev. D65 (2002) 125006 [arXiv:hep-ph/0202194].

[10] D. Zwanziger, Phys. Rev. D65 (2002) 094039 [arXiv:hep-th/0109224]. 
[11] J. M. Pawlowski, D. F. Litim, S. Nedelko, L. von Smekal, Phys. Rev. Lett. 93 (2004) 152002 |arXiv: hep-th/0312324.

[12] R. Alkofer and L. von Smekal, Phys. Rept. 353 (2001) 281 arXiv:hep-ph/0007355.

[13] R. Alkofer, L. von Smekal, Nucl. Phys. A680 (2000) 133 arXiv:hep-ph/0004141]; R. Alkofer, L. von Smekal, P. Watson, arXiv: hep-ph/0105142.

[14] L. von Smekal, arXiv:0812.0654 [hep-th]

[15] J. C. Taylor, Nucl. Phys. B33 (1971) 436.

[16] P. Boucaud, et al., arXiv:hep-ph/0507104

[17] P. Boucaud, et al., JHEP 06 (2008) 012 |arXiv:0801.2721|.

[18] W. Celmaster, R. J. Gonsalves, Phys. Rev. D20 (1979) 1420.

[19] P. Boucaud, et al., Phys. Rev. D79 (2009) 014508 [arXiv:0811.2059|.

[20] K. G. Chetyrkin, T. Seidensticker, Phys. Lett. B495 (2000) 74 arXiv: hep-ph/0008094].

[21] K. G. Chetyrkin, A. Retey, arXiv: hep-ph/0007088

[22] C. S. Fischer, J. Phys. G 32 (2006) R253 larXiv: hep-ph/0605173].

[23] O. V. Tarasov, A. A. Vladimirov, Sov. J. Nucl. Phys. 25 (1977) 585; JINRE2-80-483; O. V. Tarasov, A. A. Vladimirov, A. Y. Zharkov, Phys. Lett. B93 (1980) 429.

[24] A. I. Davydychev, P. Osland, O. V. Tarasov, Phys. Rev. D58 (1998) 036007 |arXiv:hep-ph/9801380); arXiv:hep-ph/9811503

[25] K. G. Chetyrkin, Nucl. Phys. B710 (2005) 499 arXiv: hep-ph/0405193.

[26] K. G. Chetyrkin, B. A. Kniehl, M. Steinhauser, Phys. Rev. Lett. 79 (1997) 2184-2187. arXiv: hep-ph/9706430

[27] T. van Ritbergen, J. A. M. Vermaseren, S. A. Larin, Phys. Lett. B400 (1997) 379 [arXiv:hep-ph/9701390]; M. Czakon, Nucl. Phys. B710 (2005) 485 [arXiv: hep-ph/0411261].

[28] M. Beneke, M. Jamin, JHEP 09 (2008) 044 |arXiv:0806.3156|.

[29] K. Maltman, T. Yavin, Phys. Rev. D78 (2008) 094020 arXiv:0807.0650.

[30] S. Narison, Phys. Lett. B673 (2009) 30-36. arXiv:0901.3823

[31] M. Davier, S. Eidelman, A. Hocker, Z. Zhang, Eur. Phys. J. C31 (2003) 503 arXiv: hep-ph/0308213.

[32] M. Davier, Nucl. Phys. Proc. Suppl. 169 (2007) 288 arXiv:hep-ph/0701163].
[33] K. Maltman, Phys. Lett. B633 (2006) 512 |arXiv:hep-ph/0504201|.

[34] G. W. Bennett, et al., Phys. Rev. Lett. 92 (2004) 161802 arXiv:hep-ex/0401008|.

[35] P. A. Baikov, K. G. Chetyrkin, J. H. Kühn, Phys. Rev. Lett. 101 (2008) 012002 arXiv:0801.1821.

[36] K. Maltman, in preparation.

[37] K. Maltman, J. Kambor, Phys. Rev. D65 (2002) 074013 |arXiv:hep-ph/0108227|; Phys. Lett. B517 (2001) 332 |arXiv:hep-ph/0107060|.

[38] M. Jamin, J. A. Oller, A. Pich, Eur. Phys. J. C24 (2002) 237 arXiv:hep-ph/0110194]; Phys. Rev. D74 (2006) 074009 [arXiv:hep-ph/0605095].

[39] K. G. Chetyrkin, A. Khodjamirian, Eur. Phys. J. C46 (2006) 721 arXiv:hep-ph/0512295].

[40] C. Becchi, S. Narison, E. de Rafael, F. J. Yndurain, Zeit. Phys. C8 (1981) 335.

[41] L. Lellouch, E. de Rafael, J. Taron, Phys. Lett. B414 (1997) 195 |arXiv:hep-ph/9707523

[42] E. Gamiz, M. Jamin, A. Pich, J. Prades, F. Schwab, JHEP 01 (2003) 060 |arXiv:hep-ph/0212230; Phys. Rev. Lett. 94 (2005) 011803 arXiv:hep-ph/0408044]; PoS KAON (2008) 008 arXiv:0709.0282|.

[43] K. Maltman, C. E. Wolfe, Phys. Lett. B639 (2006) 283 |arXiv:hep-ph/0603215|; Phys. Lett. B650 (2007) 27 arXiv:hep-ph/0701037;; arXiv:hep-ph/0703314

[44] A. Pich, Nucl. Phys. Proc. Suppl. 181 (2008) 300 |arXiv:0806.2793.

[45] K. Maltman, C. E. Wolfe, S. Banerjee, J. M. Roney, I. Nugent, Int. J. Mod. Phys. A23 (2008) 3191 arXiv:0807.3195|.

[46] K. Maltman, Phys. Rev. D58 (1998) 093015 |arXiv: hep-ph/9804298; K. Maltman, J. Kambor, Phys. Rev. D64 (2001) 093014 arXiv:hep-ph/0107187].

[47] P. A. Baikov, K. G. Chetyrkin, J. H. Kühn, Phys. Rev. Lett. 95 (2005) 012003 arXiv:hep-ph/0412350|. 